\title{
Collisional model of the drag force of granular impact
}

\author{
Cacey Stevens Bester ${ }^{1, \star}$ and Robert $P$. Behringer ${ }^{1, \star \star}$ \\ ${ }^{1}$ Department of Physics, Duke University, Durham, NC 27708, USA
}

\begin{abstract}
A dense, dry granular target can cause a free-falling intruding object to come to an abrupt stop as its momentum is lost to the grains. An empirical force law describes this process, characterizing the stopping force as the sum of depth-dependent friction and velocity-dependent inertial drag. However, a complete interpretation of the stopping force, incorporating grain-scale interactions during impact, remains unresolved. Here, the momentum transfer is proposed to occur through sporadic, normal collisions with clusters of high force-carrying grains at the intruder's surface. To test this model in impact experiments, we determine the forces acting on an intruder decelerating through a dense granular medium using high-speed imaging of its trajectory. We vary the geometry of the impacting object to infer intruder-grain interactions. As a result, we connect the inertial drag to the effect of intruder shape based on the proposed collisional model. These impact studies serve as an approach to understand dynamic force transmission in granular media.
\end{abstract}

\section{Introduction}

Mechanical probing by way of impact serves as a central method to explore momentum transfer in granular media. For instance, when a solid sphere is dropped into dry granular material, the intruder penetrates into the medium and suddenly decelerates to rest. The drag force from granular media is then studied through impact. There is long, rich history of the study of granular impact $[1,2]$ as well as a natural connection to planetary-scale impact phenomena [3-7]. However, a complete understanding of the physics of granular impact is still lacking.

An empirical force law has been proposed to describe the deceleration $\ddot{z}$ due to the granular target [8]. It compares the force of gravity $m g$ with the velocity-dependent inertial drag $h(z) \dot{z}^{2}$ and depth-dependent friction force $f(z)$ as

$$
m \ddot{z}=m g-h(z) \dot{z}^{2}-f(z)
$$

The inertial drag governs much of intruder dynamics during deceleration, and its $\dot{z}^{2}$ dependence suggests a collisional process. Yet the grain-scale origin of the inertial drag force is not fully determined.

Force transmission of granular impact was recently explored via experiments of intruders impacting a twodimensional arrangement of photoelastic grains [9], allowing for imaging of the granular response. This reveals a dynamic force network propagating through the granular target. Deceleration of the intruding object was found to be dominated by sporadic collisions with high force carrying clusters. These force chains propagate perpendicular from the intruder surface, indicating that intruder-

\footnotetext{
^e-mail: cacey.stevens@phy.duke.edu
}

${ }^{\star}$ e-mail: bob@phy.duke.edu grain friction is not important for initial deceleration, when $h(z) \dot{z}^{2}$ dominates. This observation of normally propagating force chains was also observed using intruders with rough and textured surfaces. The inertial drag force of granular media can therefore be described by a collisional model [10].

Here we extend to three-dimensional impact experiments and connect the inertial drag of granular impact to its grain-scale origin. We use high-speed imaging to determine the forces acting on an intruder during impact. As we vary the conical shape of the intruder, its resultant trajectory changes and can be connected to force propagation occurring perpendicular to the surface. As a result, we develop a $3 \mathrm{D}$ collisional-based description which is consistent with inertial drag values.

\section{Tracking intruder dynamics}

A schematic of the experiment setup is displayed in fig. 1(a). In each experimental run, an intruder impacts a granular target of dry, noncohesive grains of either sand or couscous, which are held within a $49 \mathrm{~cm}$ length $\mathrm{X} 38$ $\mathrm{cm}$ width $\mathrm{X} 20 \mathrm{~cm}$ depth container. The granular bed is large enough to minimize interactions with the container boundary [11], and the packing fraction, the fraction of space that is filled by grains, is held about constant. Before each run, the container is tapped then the granular bed is leveled using a straight edge. The intruder is dropped from a height of up to $2 \mathrm{~m}$, falls under gravity, and impacts the granular target. A thin rod is attached to the intruder for tracking during penetration in the grains.

We methodically vary the intruder-grain interactions during penetration by using cone-shaped intruders. An example is depicted in fig. 1(b), which shows an illustra- 
(a)

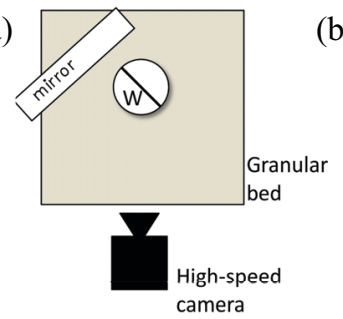

(c)

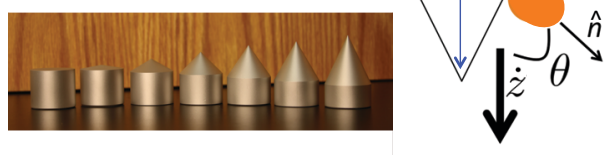

Figure 1. (a) Top view of experiment setup. A mirror is placed at 45 degrees to the impact to measure any tilting during penetration. (b) Diagram of the impact of a conical intruder with a chain of particles within the granular target (orange ellipse). (c) Image of intruders of $w=3.8 \mathrm{~cm}$, varying $s$.

tion of a conical intruder colliding with a single cluster of grains (represented by an orange ellipse). At impact, force is expected to propagate perpendicular to the surface with $\theta$ being the angle between the intruder's velocity $\dot{z}$ and surface normal $n$. Cone shapes are defined by its slope $s$, the ratio of the length of the cone $l_{\text {tip }}$ to its width $w$, and ranges from 0 (cylinder) to 2.1 (a sharp cone) (see fig. 1(c)). With increasing $s$, we thereby increase $\theta$ of expected force propagation. The effect of the cone width $w$ is also explored for $w=1.9 \mathrm{~cm}$ to $w=3.8 \mathrm{~cm}$ of $s=1$ cones. The mass of all intruders is held constant at $101 \pm 2 \mathrm{~g}$.

We place a Photron Fastcam SA5 high-speed camera at a side view of the granular bed to record the results. Videos are captured at 30,000 frames per second as the intruder comes to rest within $50 \mathrm{~ms}$. From each frame, we track the position $z$ of the intruder with an accuracy of about 0.04 $\mathrm{cm}$ (1 pixel). We then differentiate $z(t)$ to measure velocity $\dot{z}$ and perform a convolution with a gaussian filter of cutoff frequency of $960 \mathrm{~Hz}$ to reduce the amplified experimental error from $z(t)$.

The dynamics of $z(t)$ and $\dot{z}(t)$ are shown in fig. 2 for cones of $s$ from 0 to 2.1 as they impact and come to rest in the granular bed of sand. From fig. 2(a), we learn that $z$ approaches and saturates at a maximum penetration depth at about $0.04 \mathrm{~s}$, with $z=0$ and $t=0$ at impact. This trend is consistent with different $s$. Intruders with higher $s$ penetrate deeper, given the same initial energy. We take the derivative of $z(t)$ to find $\dot{z}(t)$ for different $s$ as given in fig. 2(b); $\dot{z}(t)$ declines less rapidly with $t$ as we increase $s$. However $\dot{z}$ reaches 0 at approximately the same time. We differentiate $\dot{z}(t)$ to find intruder acceleration $\ddot{z}(t)$, which reflects forces exerted by the granular medium. Forces are accordingly determined by high-speed imaging of the paths $z(t)$.

\section{3D Collisional model}

\subsection{Description}

We use the change of impact motion from different intruder shapes to verify that normal collisions capture in-
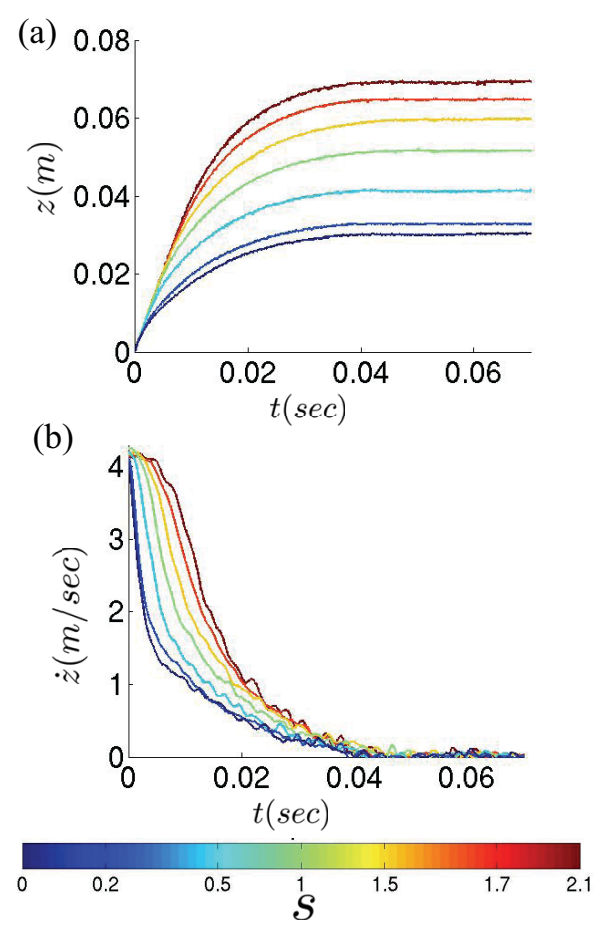

Figure 2. (a) $z$ vs. $t$, varying $s$, all impacting sand from the same release height. The maximum penetration depth increases with increasing $s$. (b) $\dot{z}$ vs. $t$. The decline of $\dot{z}(t)$ changes with increasing $s$, but all curves reach $\dot{z}=0$ at approximately the same time. Data sets correspond to $s$ values given by the colorbar.

ertial drag. Since momentum is lost by collisions perpendicular to the surface (fig. 1(b)), different inertial drag values are expected to arise with changing $s$. Accordingly, we propose an explanation of $h(z) \dot{z}^{2}$ as a collisional-based model in terms of the intruder shape.

The force due to an intruder-grain collision can be expressed as the ratio of momentum $\Delta p=m_{g} \dot{z} \cos \theta$ to time $\Delta t=d / \dot{z} \cos \theta$, where $m_{g}$ and $d$ are the grain mass and diameter, respectively. By assuming these collisions are equally likely across the surface $d S$, the number of discrete collisions is then given by $d S \cos / d^{2}$, and total force is calculated by integrating over the surface area. Forces due to collisions along the intruder surface can consequently be expressed as

$$
h(z) \dot{z}^{2}=\int \frac{\Delta p}{\Delta t} d S \propto \rho_{g} w^{2} \dot{z}^{2} \cos ^{2} \theta
$$

where $\rho_{g}$ is the grain density. The shape effect due to inertial drag is indicated through $w$ and $\cos \theta$. With geometric arguments, we can express eq. 2 in terms of $s$ as

$$
h(z) \dot{z}^{2} \propto \frac{\rho_{g} w^{2} \dot{z}^{2}}{1+s^{2}}
$$

We thereby connect the inertial drag of the macroscopic force law to a simple scaling relation that depends on intruder shape ( $s$ and $w$ ) and $\rho_{g}$, and we show consistency of $h(z) \dot{z}^{2}$ measurements with changes to $s, w$, and $\rho_{g}$.

We experimentally determine $h(z) \dot{z}^{2}$ to demonstrate that eq. 3 connects to the grain-scale origin of inertial drag of 3D granular impact. Using the force law to find 
$h(z)$ would require finding $z, \dot{z}$ and $\ddot{z}$ for many trajectories of each intruder. Additionally, acceleration data $\ddot{z}$ can present significant error due to filtering [12]. Accordingly, previous authors have shown that the differential equation can be solved by reframing in the kinetic energy $K$ versus $z$ form where $K=1 / 2 m \dot{z}^{2}$ and $m \ddot{z}=d K / d z[13,14]$. The approach leads to the following:

$$
\frac{d K}{d z}=m g-f(z)-\frac{2 h(z)}{m} K
$$

Therefore, we acquire $h(z)$ from $z$ and $\dot{z}$ and achieve

$$
K(z)=e^{-\int \frac{2 h(z)}{m} d z}\left[K_{i}+\int(m g-f(z)) e^{\int \frac{2 h(z)}{m} d z} d z\right]
$$

when eq. 4 is solved with the integrating factor $e^{\int \frac{2 h(z)}{m} d z}$.
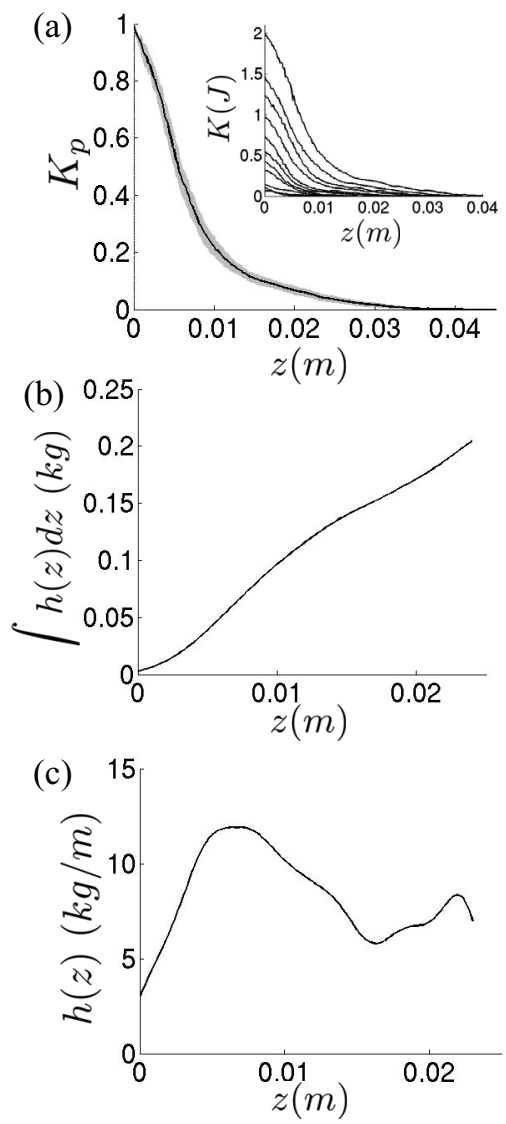

Figure 3. (a) Inset: $K$ versus $z$ of $s=0$ intruder impacting sand. Each curve represents a trajectory of a different $\dot{z}_{i}$. Main panel: $K_{p, a v g}$ versus $z$, as determined from all pairs of $K(z)$ trajectories of the inset plot. The gray region surrounding the curve indicates the standard deviation from the mean of $K_{p}$. (b) $\int h(z) d z$ versus $z$ (c) $h(z)$ versus $z$, showing oscillations about a constant value.

We take the difference of two $K(z)$ curves from experimental runs with distinct impact velocity $\dot{z}_{i}$ to isolate $h(z)$ and obtain the relation

$$
K_{p}=\frac{K_{b}-K_{a}}{K_{b, i}-K_{a, i}}=e^{-\int \frac{2 h(z)}{m} d z}
$$

where $a$ and $b$ indicate indices of different trajectories. By averaging all pairs of $K(z)$ trajectories for a range of $\dot{z}_{i}$, $h(z)$ is found for each intruder.
Figure 3 shows how we determine $h(z)$ for an $s=0$ intruder penetrating sand. We start by expressing data as $K(z)$, as shown in the inset of fig. 3(a). There is a rapid decline of $K$ during penetration. The average of differences of all pairs of $K(z)$ trajectories is then determined (see fig. 3(a)), and we use eq. 5 to solve for $\int h(z) d z$, given in fig. 3(b). The solution in this case is a $\int h(z) d z$ curve that is a linear function of $z$. The derivative of this curve gives $h(z)$ and requires noise reduction by performing a convolution to the data (see fig. 3(c) for result). There are oscillations in $h(z)$ which may connect to the force fluctuations and discretization of collisions [9]; a precise determination of these features would require direct force measurement. To avoid handling noise amplification and possible data distortion introduced by taking derivative of $\int h(z) d z$, we utilize $\int h(z) d z$ for the $s, w$, and $\rho_{g}$ comparisons.

\subsection{Intruder shape and grain density}

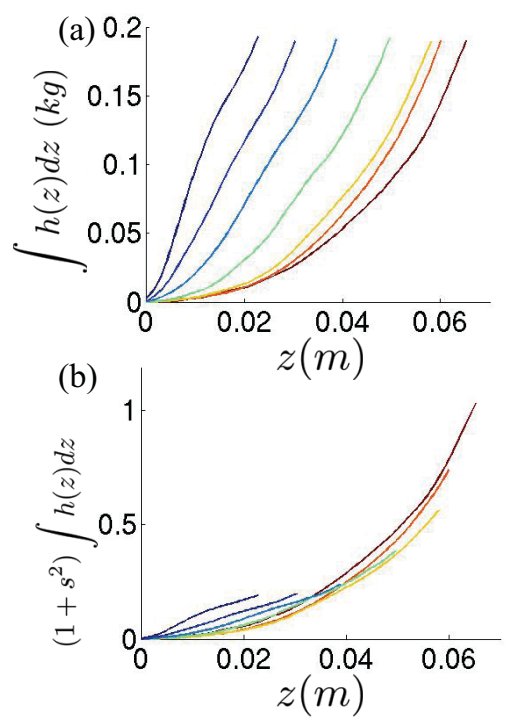

Figure 4. (a) $\int h(z) d z$ versus $z$ for all intruders, ranging from $s=0$ to $s=2.1$. Colors correspond with $s$ values of fig. 2. Higher $s$ lowers the curve to smaller inertial drag values. (b) The data collapse to one curve when scaled as a function of $s$.

Starting with the shape effect, we show that $s$ and $w$ affect $h(z)$ as described by eq.3. In fig. 4(a), $\int h(z) d z$ versus $z$ is plotted as $s$ ranges from 0 to 2.1. Curves shift to lower inertial drag values with increasing $s$. We also find that $\int h(z) d z$ vs. $z$ plots become increasingly curved as $s$ increases. We propose that the increased curvature results from the changing surface area at low $z$ as a sharper cone is penetrated deeper into the granular target. Since $w$ is increasing at $z=0$ up to $z=l_{\text {tip }}$ and $\int h(z) d z$ is proportional to $w^{2}, \int h(z) d z$ rises quadratically in this range. After $z>l_{\text {tip }}$, $\int h(z) d z$ becomes a linear function of $z$.

All curves fall onto a single curve when the $\int h(z) d z$ axis is rescaled by a function of $s$, as shown in fig. 4(b). The expression $1+s^{2}$ leads to a collapse of all data as indeed shown in eq. 3. The scaling therefore shows that the collisional model does agree with our 3D experiments. 


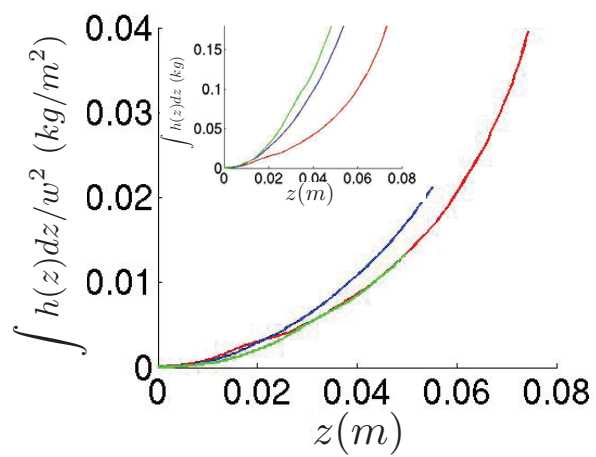

Figure 5. Inset: $\int h(z) d z$ versus $z$ of $s=1$ intruder, varying width $w: 1.9 \mathrm{~cm}, 3.0 \mathrm{~cm}$, and $3.8 \mathrm{~cm}$. Lower $w$ decreases the curve, but, in this case, the trend in data is not affected. Main panel: When scaled as a function of $w$, the data collapse to a single curve.

When extending the study to the effect of $w$, we similarly observe that the collisional model applies. Figure 5 shows $\int h(z) d z$ as a function of $z$ for $s=1$ cones of three widths: $w=1.9 \mathrm{~cm}, w=3.0 \mathrm{~cm}$, and $w=3.8 \mathrm{~cm}$. We see that $\int h(z) d z$ decreases with decreasing $w$ as displayed in the inset plot. Additionally, the curved-linear transition of each curve connects with $l_{\text {tip }}$. The dependence of inertial drag on $w$ is then displayed by a collapse of data as $\int h(z) d z \propto w^{2}$. These data rescale to a single curve when plotted as $\int h(z) d z / w^{2}$.

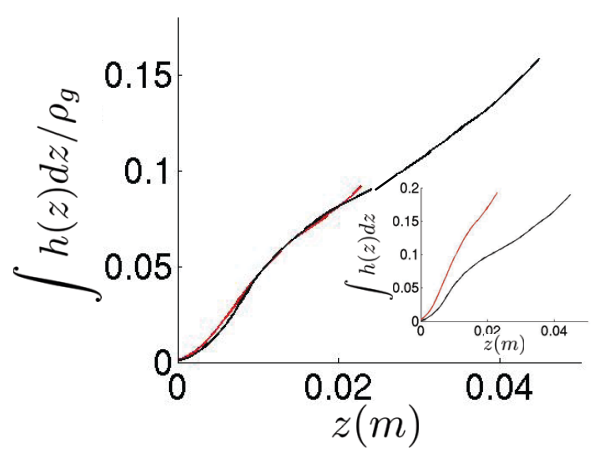

Figure 6. Inset: $\int h(z) d z$ versus $z$ for $s=0$ intruder, varying $\rho_{g}$ : sand (red line) or couscous (black line). Higher $\rho_{g}$ (sand) increases inertial drag coefficient $h(z)$. Main panel: Scaled $h(z)$, $\int h(z) d z / \rho_{g}$ versus $z$ for the two grains, collapsing data.

Lastly, we vary the granular target (sand or couscous) to study the effect of grain density $\rho_{g}$. Both targets are prepared by pouring grains into the container and tapping to settle the surface. The inertial drag coefficient $h(z)$ is determined for an $s=0$ intruder impacting the sand or couscous target. For $s=0, \int h(z) d z$ is a linear function of $z$. We show that our collisional model also captures the effect of $\rho_{g}$ (see Fig. 6). There is a shift of $h(z)$ with $\rho_{g}$; we find that $\int h(z) d z$ is larger for higher $\rho_{g}$, as expected from the $h(z)$ expression of the collisional model (eq. 3). Ac- cordingly, there is a higher stopping force due to sand, and this affects the maximum penetration depth of the intruder. The two curves collapse to a single curve, when scaled by $\rho_{g}$ as ascribed in the collisional model.

\section{Conclusions}

We study the dynamics of a cone-shaped intruder impacting with dry granular media. Granular impact is described, in part, by a velocity-dependent inertial drag force. Based on its dependence on intruder shape, we connect the inertial drag from the granular target to sporadic, normal collisions with force-carrying chains of grains. A collisional model is presented to support our description of inertial drag for 3D impact and is explored through our experimental data. The collapse of the inertial drag coefficient when accounting for intruder shape and granular target demonstrates the validity of the model and shows that intrudergrain friction can be ignored for this term. These scaling results are consistent with our observation of dynamic force transmission in granular media.

\section{Acknowledgements}

This work has been supported by Duke University Provost's Postdoctoral Program, NSF-DMR1206351, and NASA grant NNX15AD38G. We appreciate the assistance from Jonathan Bares, Hu Zheng, and Yuchen Zhao.

\section{References}

[1] J. Poncelet, Cours de Mecanique Industrielle (1829)

[2] H. Katsuragi, Physics of Soft Impact and Cratering (Springer, Tokyo, 2016)

[3] C. Li, T. Zhang, D. Goldman, Science 339, 1408 (2013)

[4] K. Daniels, J. Coppock, R. Behringer, Chaos 14, S4 (2004)

[5] C. Guttler, N. Hirata, A. Nakamura, Icarus 220, 1040 (2012)

[6] D. Dowling, T. Dowling, Am. J. Phys. 81, 875 (2013)

[7] J. Ruiz-Suarez, Rep. Prog. Phys. 76, 066601 (2013)

[8] H. Katsuragi, D. Durian, Nat. Phys. 3, 420 (2007)

[9] A. Clark, L. Kondic, R. Behringer, Phys. Rev. Lett. 109, 238302 (2012)

[10] A. Clark, A. Petersen, R. Behringer, Phys. Rev. E 89, 012201 (2014)

[11] A. Seguin, Y. Bertho, P. Gondret, Phys. Rev. E 78, $010301(2008)$

[12] D.M. Meko, Applied Time Series Analysis (UA Press, 2015)

[13] M.A. Ambroso, R.D. Kamien, D.J. Durian, Phys. Rev. E 72, 041305 (2005)

[14] A.H. Clark, R.P. Behringer, EPL 101, 64001 (2013) 